\title{
Uma experiência exitosa de meio século da Universidade de São Paulo junto à comunidade
}

\author{
A successful experience of half a century of the University of São Paulo with \\ the community
}

Ivan Savioli Ferraz ${ }^{1}$, Luiz Antonio Del Ciampo ${ }^{1}$

\begin{abstract}
RESUMO
A extensão constitui-se em parte indissociável das atividades universitárias e elo fundamental da interface da academia com a comunidade. A participação da universidade na assistência à saúde da comunidade talvez represente uma das faces mais visíveis e, ao mesmo tempo, sensíveis da extensão universitária. A atuação da Universidade de São Paulo (USP) no Centro Médico Social Comunitário "Vila Lobato" (CMSC "Vila Lobato") tem se constituído por uma experiência exitosa de meio século junto à comunidade servida por esta unidade básica de saúde de Ribeirão Preto. Alunos de graduação do curso de medicina e de nutrição e metabolismo da Faculdade de Medicina de Ribeirão Preto-USP (FMRP-USP) e, também, médicos residentes do Hospital das Clínicas da FMRP-USP têm prestado assistência à saúde à população local. Alunos de graduação de outras unidades do Campus de Ribeirão Preto da USP (odontologia, enfermagem e psicologia) também atuam no CMSC "Vila Lobato". Inúmeros projetos de pesquisa na área da saúde foram e continuam sendo desenvolvidos na unidade, beneficiando a comunidade. A experiência de meio século da USP junto ao CMSC "Vila Lobato" constitui-se por modelo bem-sucedido de extensão universitária, devendo não somente ser reconhecido de forma genuína no meio acadêmico, mas também estimulada.
\end{abstract}

Palavras-chave: Extensão Comunitária. Universidades. Atenção Primária à Saúde. Ensino. Pesquisa.

\begin{abstract}
The university extension program is inseparable from the university activities and also an essential connection between the academia and the community. The participation of the university in health care to the community may represent one of the most visible and at the same time sensitive aspects of university extension. The work of the University of São Paulo (USP) at the Community Social Medical Center "Vila Lobato" (CSMC "Vila Lobato") has been a successful experience of half a century in the community assisted by this primary health care unit of Ribeirão Preto. Undergraduate students of both Medicine and Nutrition and Metabolism courses from Ribeirão Preto Medical School-USP (RPMS-
\end{abstract}

1. Docente do Departamento de Puericultura e Pediatria, Faculdade de Medicina de Ribeirão Preto da USP.
CoRRESPONDÊNCIA: Luiz Antonio Del Ciampo Departamento de Puericultura e Pediatria, Faculdade de Medicina de Ribeirão Preto da USP. Avenida Bandeirantes, 3900 14049-900 - Ribeirão Preto/SP - Brasil delciamp@fmrp.usp.br

Recebido em 04/09/2018 Aprovado em 27/03/2019 
USP) and also resident physicians from the General Hospital of RPMS-USP have provided health care to the local population. Undergraduate students from other schools from USP at Ribeirão Preto (dentistry, nursing, and psychology) have also worked at the CSMC "Vila Lobato". Several research projects on health were carried out, and many others have been developed in the unit, benefiting the community. This experience of half a century of USP with the CSMC "Vila Lobato" is a successful model of university extension, and it should be recognized genuinely in the academic institutions and also stimulated.

Keywords: Community-Institutional Relations. Universities. Primary Health Care. Teaching. Research.

\section{INTRODUÇÃO}

A universidade como instituição comprometida com a produção e difusão de conhecimentos e saberes científicos tem sua estrutura básica caracterizada por atividades desenvolvidas no trinômio ensino, pesquisa e extensão. ${ }^{1} \mathrm{~A}$ extensão universitária, por sua vez, é um processo educativo e científico que integra o ensino e a pesquisa com as necessidades da comunidade, reunindo saberes e competências de naturezas diferentes, que se completam em busca de objetivos comuns. Nesse contexto, pode ser considerada como uma ponte permanente entre a universidade e os diversos setores da sociedade. A universidade leva à comunidade, através de projetos, os conhecimentos originados de suas pesquisas com a finalidade de modificar a realidade social, produzir transformações e democratizar a participação popular. ${ }^{2}$ Por sua vez, a sociedade recebe benefícios diretos e indiretos, visto que esses projetos Ihe direcionam oportunidades de desenvolvimento cultural, social e política, ao contemplarem as demandas que a comunidade necessita.

$\mathrm{O}$ que surgiu como atividade quase que filantrópica no período medieval passa a ser reconhecido, a partir de 1871, em Cambridge, na Inglaterra, como uma forma de integrar a universidade no cotidiano da sociedade. No Brasil, a extensão surge a partir de 1931, quando foi elaborado o primeiro estatuto das universidades brasileiras. ${ }^{3}$ Desde então, tem sido implantada por inúmeras instituições universitárias, aperfeiçoando as formas de aproximação e trabalho conjunto com a sociedade.

Nessas atividades de extensão o estudante tem a oportunidade de sair do seu cotidiano no ambiente universitário e se inserir na comunidade para observar as relações sociais, seus problemas e propor a busca de soluções, atuando como protagonista de sua formação técnica à medida que obtém as competências necessárias à atuação profissional. Além disso, age como cidadão ao integrar-se e reconhecer-se como agente da garantia dos direitos e deveres, assumindo compromissos e adotando ações que possam transformar aquela comunidade. . $^{4}$

Mais especificamente no que diz respeito aos cursos das áreas de ciências da saúde destaca-se a oportunidade de os estudantes serem integrados à rede assistencial primária e secundária locais, que servem de espaço para novas experiências direcionadas ao cuidado pessoal, à humanização e à qualificação da atenção à saúde; desta forma, a extensão universitária na área da saúde se apresenta como oportunidade ímpar para alunos e docentes conhecerem e atuarem em cenários e situações concretas de trabalho dentro do Sistema Único de Saúde (SUS). ${ }^{6,7}$ É o momento de atuar em práticas mais sociais, direcionando suas ações aos indivíduos, sejam educativas ou melhorando a eficiência dos serviços com maior participação e desprendimento. Ao mesmo tempo, a sociedade também se torna agente de transformação a partir do momento que direciona à universidade todos os seus recursos e demandas, em um movimento bidirecional que tende a beneficiar todos os partícipes. , $^{8,9}$

Além das características de ensino e de assistência ou extensão de serviços, deve-se também identificar as possibilidades de desenvolvimento de projetos de pesquisa, com base nas demandas locais e regionais, que atendam tanto às expectativas da universidade quanto as necessidades da sociedade, compreendendo, desse modo, que o processo educação-extensão-pesquisa, indissociável por natureza, deva sempre se fazer presente. ${ }^{10}$ 
A propósito dessas importantes considerações acerca da relevância dessa inerente atividade acadêmica em particular, este artigo apresenta e analisa uma experiência bem-sucedida no campo da extensão universitária na área da saúde implantada pela Universidade de São Paulo (USP), na cidade de Ribeirão Preto.

\section{Os primeiros anos da extensão universitária} na Faculdade de Medicina de Ribeirão da Universidade de São Paulo

Na década de 1950, nos primeiros anos de funcionamento da Faculdade de Medicina de Ribeirão Preto da Universidade de São Paulo (FMRP-USP), o Prof. Dr. José Lima Pedreira de Freitas já propunha a implantação de um centro de saúde fora do espaço físico universitário. O local escolhido para a instalação desse projeto pioneiro ficava a $80 \mathrm{~km}$ da sede da FMRP-USP, mais precisamente no município paulista de Cássia dos Coqueiros, cidade na qual o Prof. Pedreira de Freitas havia desenvolvido estudos de campo sobre epidemiologia e controle de doença de Chagas. ${ }^{11,12} \mathrm{O}$ fundador e primeiro diretor da FMRP-USP, Prof. Dr. Zeferino Vaz, que também vislumbrava a possibilidade de levar os alunos do curso de graduação em medicina para fora dos muros do campus universitário, aprovou a ideia do Prof. Pedreira de Freitas ${ }^{13}$ e, apesar de não ter obtido sucesso nesse propósito durante o período que esteve à frente da direção da FMRP-USP, a semente havia sido lançada.

Dessa forma, seguindo os anseios do Prof. Pedreira de Freitas, primeiro chefe do pioneiro Departamento de Higiene e Medicina Preventiva (hoje, Departamento de Medicina Social) da FMRP-USP, em 1964 era instalado o Instituto de Medicina Rural no município de Cássia dos Coqueiros. ${ }^{14}$

No final da década de 1950 e durante toda a década de 1960, alguns docentes da FMRP-USP retomaram a ideia e desencadearam várias iniciativas para levar, literalmente, a universidade ao encontro da comunidade. ${ }^{12} \mathrm{Em} 1969$ era criado o Centro Médico Social Comunitário "Vila Lobato" (CMSC "Vila Lobato") no município de Ribeirão Preto e em 1971, a FMRP-USP, através de seu Departamento de Puericultura e Pediatria, iniciava as suas atividades no Centro Médico Social Comunitário de Pradópolis, na cidade paulista de mesmo nome, distante $38 \mathrm{~km}$ de Ribeirão Preto. A profícua vivência em Pradópolis estendeu-se até o ano de $1991 .^{13}$ Algumas dessas experiências, entretanto, permanecem até hoje.

\section{Uma experiência exitosa de cinco décadas}

Como citado anteriormente, dentre as iniciativas de estender as atividades da universidade para fora de seus muros estava a da implantação de um centro de saúde em um bairro periférico da cidade de Ribeirão Preto, que à época contava com poucos recursos oferecidos à comunidade. Com o empenho junto à Secretaria Municipal da Saúde de Ribeirão Preto (SMSRP) do Prof. Dr. Jacob Renato Woiski, fundador e primeiro chefe do Departamento de Puericultura e Pediatria da FMRP-USP, e contando com o apoio dos Departamentos de Ginecologia e Obstetrícia e de Higiene e Medicina Preventiva, foram iniciadas as atividades do CMSC "Vila Lobato" no ano de 1969. Essa unidade de saúde foi construída em um bairro pouco desenvolvido, sem saneamento básico, situado a meio caminho entre o centro da cidade e o campus universitário. Estava implantada aí a primeira unidade situada fora do âmbito tradicional - ou seja, dentro dos limites do campus universitário - no município de Ribeirão Preto. ${ }^{13,14}$

No início, alunos de graduação e médicos residentes das áreas de pediatria, clínica médica, medicina social, psiquiatria e ginecologia e obstetrícia estagiavam no local sob a supervisão dos docentes da FMRP-USP de cada um dos respectivos Departamentos. Deve ser ressaltado que a Escola de Enfermagem de Ribeirão Preto da Universidade de São Paulo (EERP-USP), por meio de sua diretora à época, Profa. Glete de Alcântara, vislumbrando o sucesso dessa iniciativa, também participou do projeto inicial. ${ }^{13}$

Porém, ao longo dos primeiros anos de funcionamento, alguns Departamentos da FMRP-USP e a EERP-USP deixaram de atuar no CMSC "Vila Lobato". Entretanto, desde o início os Departamentos de Puericultura e Pediatria e Ginecologia e Obstetrícia sempre estiveram presentes na unidade, e assim continuam até o presente momento. 
Em 2015, após uma reforma que possibilitou a ampliação física do prédio do CMSC "Vila Lobato", o Departamento de Clínica Médica da FMRP-USP retornou com suas atividades acadêmicas (ensino e pesquisa) e assistenciais na unidade.

Atualmente o CMSC "Vila Lobato" é uma unidade básica de saúde integrada à rede assistencial do município de Ribeirão Preto e está plenamente inserida no SUS. Seu funcionamento é definido por um regimento elaborado pela FMRP-USP (que indica o docente médico gerente da unidade) em convênio com a SMSRP, Hospital das Clínicas da FMRP-USP (HC-FMRP-USP) e Fundação de Apoio ao Ensino, Pesquisa e Assistência (FAEPA) do HC-FMRP-USP. De acordo com dados da Divisão de Planejamento em Saúde da Secretaria Municipal da Saúde de Ribeirão Preto, com base no censo populacional do ano de 2010 do Instituto Brasileiro de Geografia e Estatística (IBGE), a população assistida pelo CMSC "Vila Lobato" está estimada em 16.000 habitantes. ${ }^{15}$

Desde o início de suas atividades, o CMSC "Vila Lobato" tem se mostrado como um profícuo campo para o desenvolvimento dos três pilares das atividades da vida universitária: ensino, pesquisa e, principalmente, extensão, oferecendo assistência à saúde da população residente naquela área.

\section{Ensino multidisciplinar e interdisciplinar}

Em relação ao ensino, alunos de terceiro, quarto, quinto e sexto anos do curso de graduação em medicina da FMRP-USP estagiam, supervisionados por docentes da faculdade e de médicos assistentes do HC-FMRP-USP, nos ambulatórios de puericultura e pediatria (incluindo, aqui, a hebiatria - atendimento aos adolescentes), ginecologia e obstetrícia e clínica médica da unidade. Além disso, alunos do curso de graduação de nutrição e metabolismo da FMRP-USP também estagiam nas dependências do CMSC "Vila Lobato" prestando assistência à população pediátrica e adolescente. Com intuito de tornar o CMSC "Vila Lobato" uma unidade não só multidisciplinar, mas também, interdisciplinar, incentivou-se a participação de algumas unidades do Campus da USP de Ribeirão Preto a iniciarem ou a aumentarem a sua participação no local. Desde 2016, alunos do último ano do curso de graduação em odontologia da Faculdade de Odontologia de Ribeirão Preto - USP (FORP-USP) também atuam na unidade, supervisionados por um dentista assistente e por um docente da referida faculdade. Recentemente, no início do ano de 2017, alunos de graduação do curso de psicologia do Departamento de Psicologia da Faculdade de Filosofia, Ciências e Letras de Ribeirão Preto - USP (FFCLRP-USP) iniciaram estágio no CMSC "Vila Lobato", atendendo pacientes oriundos dos ambulatórios de pediatria e puericultura da unidade. Ainda em 2017, a EERP-USP voltou a se inserir nas atividades da unidade com a participação de alunos dos últimos anos do curso de graduação em enfermagem.

Com o intuito de integrar as diversas áreas do conhecimento que atuam na unidade foram iniciadas, no mês de abril de 2017, reuniões mensais entre os graduandos de medicina, odontologia, enfermagem e psicologia para discussão de casos clínicos pediátricos com abordagem inter e multidisciplinar, sendo esses encontros coordenados pelos docentes das respectivas especialidades.

Para o ano de 2019 planeja-se a inserção da Escola de Educação Física e Esportes de Ribeirão Preto da Universidade de São Paulo (EEFERP-USP) e do Departamento de Música da FFCLRP-USP para também iniciarem atividades acadêmicas e assistenciais junto à população assistida pelo CMSC "Vila Lobato", aumentando, dessa forma, a multi e a interdisciplinaridade dentro da unidade, beneficiando não somente os alunos de graduação, mas, principalmente, a comunidade.

\section{Meío século de pesquisa}

Em relação à pesquisa, o CMSC "Vila Lobato" apresenta-se como um campo ilimitado de pesquisas utilizado por docentes e alunos de pós- graduação da FMRP-USP, permitindo a realização de inúmeras dissertações de mestrado e teses de doutorado e, consequentemente, propiciando a produção de numerosas publicações advindas desses trabalhos. Atualmente, como vem ocorrendo ao longo de seus 50 anos de existência, a unidade tem sido utilizada de forma proficiente por vários Departamentos da FMRP-USP e, também, por outras escolas do Campus da USP de Ri- 
beirão Preto para a realização de pesquisas, com mais de uma dezena de projetos em andamento, todos trazendo benefícios à comunidade servida pelo CMSC "Vila Lobato" de forma direta a curto, médio e longo prazo.

\section{Assistência à população}

Em relação à assistência, médicos residentes do HCFMRP-USP dos Programas de Pediatria, Ginecologia e Obstetrícia, Clínica Médica e Medicina de Família e Comunidade, além dos internos do curso de graduação em medicina da FMRP-USP, sob a supervisão de docentes de vários Departamentos da referida escola médica e de médicos assistentes do HC-FMRP-USP, realizam atendimento em regime ambulatorial nas três especialidades médicas (pediatria, clínica médica e ginecologia e obstetrícia) oferecido no CMSC "Vila Lobato". Acrescenta-se à assistência médica, os atendimentos odontológicos, psicológicos, enfermagem e de nutrição. Somando-se as consultas médicas, odontológicas e de enfermagem, além dos pequenos procedimentos (por exemplo: curativos, retiradas de pontos cirúrgicos, etc.), o CMSC "Vila Lobato" realiza mais de 35 mil atendimentos ao ano.

Em relação aos atendimentos pediátricos, vale ressaltar que a unidade testemunha um fenômeno interessante e, ao mesmo tempo, cativante: a assistência à saúde à segunda e terceira gerações de pacientes de uma mesma família. Tal fenômeno, infelizmente raro na saúde pública brasileira, traz benefícios não somente ao paciente e a sua família, mas também, ao profissional de saúde; ainda, a observação dessa realidade fornece subsídios para que alunos e professores reflitam sobre a questão e possam discutir sobre modelos de assistência à saúde, adequados e factíveis.

Finalmente, por meio de seu setor de Serviço Social, o CMSC "Vila Lobato" promove a realização na unidade de cursos de curta duração para os moradores da comunidade (por exemplo: cursos de pintura em tela e de corte e costura). Além disso, após a reforma e ampliação do prédio ocorrida há quatro anos, tem se tornado frequente a cessão do espaço físico da unidade à comunidade para a realização de vivências diversas tais como palestras, grupos de discussão e promoção de atividade física. Essas atividades são, em sua maioria, coordenadas por profissionais que atuam no CMSC "Vila Lobato", principalmente, assistentes sociais e médicos.

\section{CONSIDERAÇÕES FINAIS}

A extensão, hoje considerada como um dos pilares fundamentais da atuação das universidades na sociedade, era de caráter quase filantrópico na sua origem, e despontou no Brasil na primeira metade do século XX. Na FMRP-USP, as experiências iniciais na extensão ocorreram nos primeiros anos após a sua fundação. Inúmeras iniciativas de levar a universidade para junto da comunidade ocorreram na década de 1960, sendo que algumas dessas experiências continuam até hoje, como é o caso do CMSC "Vila Lobato", uma unidade básica de saúde na periferia do município de Ribeirão Preto, criado no ano de 1969. Ao longo dos anos essa atividade se expandiu para outras unidades do Campus da USP em Ribeirão Preto promovendo maior contato com a comunidade. Ao lado da assistência - sempre ligada ao ensino -, essa vivência permitiu o desenvolvimento de pesquisas que beneficiaram a comunidade assistida pela unidade de saúde.

Desta forma, entende-se que a atuação da universidade junto ao CMSC "Vila Lobato" e a sua comunidade pode servir de modelo para outras experiências bem-sucedidas de extensão na área da saúde. Tais vivências entre a universidade e a comunidade devem ser não somente reconhecidas de forma genuína no meio acadêmico, mas, acima de tudo, estimuladas.

\section{REFERÊNCIAS}

1. Santos AO, Svartman BP, Rocco FVC, Galeão-Silva LG, Casco R, Massola GM. Aproximando universidade e escola técnica: lições aprendidas com o incentivo à pesquisa no ensino médio profissionalizante. Rev. Cult. Ext. USP, São Paulo. 2016; 5: 61-73.

2. Silva $O .0$ que é extensão universitária. Integração: ensino, pesquisa e extensão. São Paulo; 1997; 3: 148-57. 
3. Pinheiro BL, Cruz IL, Chesani FH. Extensão universitária: os estilos de pensamento na área da saúde no Brasil. Rev Ed Popular. 2016; 15: 91-106.

4. Conchão S. Extensão universitária na Faculdade de Medicina do $A B C$ : quais avanços e limites? ABCS Health Sci. 2015; 40:318-23.

5. Corrêa EJ, Alves MTSSB, Santos MFH, Bernardes L, Barros RMF, Crisóstimo AL. A área da saúde e a implementação da política nacional de extensão. Rev Bras Extensão Universitária. 2006; 4: 7-13.

6. Biscarde DGS, Pereira-Santos M, Silva LB. Formação em saúde, extensão universitária e Sistema Único de Saúde (SUS): conexões necessárias entre conhecimento e intervenção centradas na realidade e repercussões no processo formativo. Interface; $2014 ; 18: 177-86$.

7. Bulcão LG. O ensino médico e os novos cenários de ensino-aprendizagem. Rev Bras Educ Méd. 2004; 28: 61-72.

8. Divino AEA, Oliveira CEL, Costa CAC, Souza Neta HR, Campos LS, Menezes RMJ et al. A extensão universitária quebrando barreiras. Aracaju, Cadernos de Graduação Ciências Humanas e Sociais. 2013; 16: 135-40.

9. Moura LFAD, Piauilino RJB, Araújo IF, Moura MS, Lima CCB, Evangelista LM, Lima MDM. Impacto de um projeto de extensão universitária na formação profissional de egressos de uma universidade pública. Rev Odontol UNESP. 2012; 41: 348-52.
10. Martins LM. A indissociabilidade Ensino-Pesquisa-Extensão como um dos fundamentos metodológicos do Ensino Superior. In Pinho SZ. (org). Oficinas de estudos pedagógicos: reflexões sobre a prática do ensino superior. São Paulo: Cultura Acadêmica: UNESP, 2008, p.102-15.

11. Franco AR, Haddad N, Forster AC, Caccia-Bava MCG. A FMRP-USP em atividades de ensino, pesquisa e extensão de serviços à comunidade, dentro do projeto Centro de Saúde-Escola. Medicina (Ribeirão Preto). 2002; 35: 425-32.

12. Mauro JEM, Nogueira AR. A Faculdade de Medicina de Ribeirão Preto - USP: primeiros tempos, através de dos documentos e pela voz de seus construtores. Ribeirão Preto, FUNPEC Editora, 2004.

13. Santoro JR. Departamento de Puericultura e Pediatria. Medicina (Ribeirão Preto). 2002; 35: 349-66.

14. Rocha JSY. Departamento de Medicina Social: a restruturação e os novos tempos. Medicina (Ribeirão Preto). 35: 306-12.

15. Ribeirão Preto. Secretaria Municipal da Saúde. Divisão de Planejamento em Saúde. Revisão das áreas de abrangência das Unidades de Saúde e Estimativa Populacional de acordo com o Censo IBGE 2010 por Unidade. Ribeirão Preto, 2011. 45 p. 